wiedergegeben ${ }^{10}$ als bei der Anpassung unter $\mathrm{Zu}$ grundelegung der $r_{0}$-Strukturdaten, was für eine $\mathrm{Ab}$ knickung der $\mathrm{CH}_{3}$-Drehachse gegen die $\mathrm{S}-\mathrm{C}$-Bindung spricht. (Der maximale mit $\lambda_{a}=0,515$ verträgliche Abknickwinkel beträgt $2^{\circ}$, d. h. er ist etwa gleich dem bei $\mathrm{CH}_{3}-\mathrm{S}-\mathrm{CH}_{3}{ }^{8,9}$ gefundenen Wert.) In Tab. 1 ist das Ergebnis dieser zweiten $s$-Anpassung nochmals zusammengefaßt. In Tab. 2 sind die bisher hochauflösend registrierten Multipletts des Schwingungsgrundzustands zusammengestellt. Die

10 Auch die Aufspaltung des $5_{14}-5_{23}$-Ubergangs wird jetzt gut wiedergegeben (vgl. Tab. 2).
Angabe berechneter Frequenz- und Aufspaltungswerte fehlt für $J>5$. Für die höheren $J$-Werte wurde die infolge der hohen Matrizenränge langwierige Rechnung bisher nur für die $\lambda$-Werte der $r_{0}$-Struktur durchgeführt, was aber, zusammen mit dem aus der Spinstatistik folgenden Intensitätsverhältnis ausreichte, um die Komponenten identifizieren zu können.

Die Deutsche Forschungsgemeinschaft hat in dankenswerter Weise die vorliegende Arbeit durch Gewährung von Sachmitteln ermöglicht.

\title{
Zur Aktivität von Methyltorsionsschwingungen in Zweikreiselmolekülen
}

\author{
H. DreizLER \\ Physikalisches Institut der Universität Freiburg i. Br. \\ (Z. Naturforschg. 22 a, 192-194 [1967] ; eingegangen am 17. November 1966)
}

\begin{abstract}
Für Moleküle mit zwei äquivalenten Methylgruppen wird ein Vorschlag formuliert, die Aktivität der Methyltorsionsschwingungen durch eine Wechselwirkung zwischen den Torsionsschwingungen und der Rotation zu verstehen. Die Wechselwirkung zwischen den beiden Methyltorsionsschwingungen ist für diese Betrachtung nicht wesentlich.
\end{abstract}

Um die Aktivität von Methyltorsionsschwingungen zu verstehen, schlug ich in einer vorangehenden Arbeit ${ }^{1}$ vor, ein Molekülmodell zu verwenden, das sich bei der Analyse der Torsionsfeinstruktur in Rotationsspektren bewährt hat. Da nur Moleküle mit einer Methylgruppe behandelt wurden, besaß das Modell drei Freiheitsgrade der äußeren Rotation und einen Freiheitsgrad der inneren Rotation (Torsion). Jeder der beiden gegeneinander tordierenden Molekülteile wurde dabei als starr vorausgesetzt. Der verwendete Hamilton-Operator in der Formulierung der PAM-Methode ${ }^{2}$ bezieht sich auf das von der Methyltorsion unbeeinflußte Hauptträgheitsachsensystem. Der Hamilton-Operator besitzt einen Wechselwirkungsanteil $H_{\mathrm{RT}}$ zwischen Torsion und äußerer Rotation. Er bewirkt die Torsionsfeinstruktur in den Rotationsspektren und, wie sich zeigte, die Aktivität der Torsionen in diesem Modell ${ }^{3}$. $H_{\mathrm{RT}}$ verursacht nämlich eine Mischung von Näherungs-

1 H. Dreizler, Z. Naturforschg. 21 a, 621 [1966].

2 C. C. Lin u. J. D. Swalen, Rev. Mod. Phys. 31, 841 [1959].

3 Streng kann man nur noch von Torsions-Rotationsübergängen sprechen. Sowohl Torsions- wie Rotationsquantenzahlen $v$ und $K_{-} K_{+}$sind nur noch genähert gute Quantenzahlen. Die Bezeichnungsweise Torsionsübergang bzw. Ro- funktionen. Sie sind das Produkt der Eigenfunktionen $U_{v \sigma}(\alpha) \cdot r_{J K_{-} K_{+}}(\psi, \vartheta, \varphi)$ des reinen Torsionsanteils $H_{\mathrm{T}}$ und des reinen Rotationsanteils $H_{\mathrm{R}}$.

Es wurde weiterhin vorgeschlagen, aber nicht ausgeführt, das starre Modell aufzugeben und Schwingungsfreiheitsgrade hinzuzunehmen. Auch in diesem Fall ist eine Aktivität durch die Mischung von Eigenfunktionen zu erwarten.

Beiden erwähnten Schemata ist gemeinsam, daß durch Mischung die Funktionen in den Torsionskoordinaten in nicht separabler Weise mit Funktionen in solchen Koordinaten verbunden werden, von denen auch der Dipolmomentoperator abhängt.

Vielfach wird das übliche Schema ${ }^{4}$ der Schwingungsspektroskopie angewendet, um Auswahlregeln für die Torsionen abzuleiten. Man verwendet aber dann die Näherung, daß die Torsionen Normalschwingungen sind. Das ist identisch mit der Annahme, daß sich das periodische (anharmonische)

tationsübergang gibt nur noch an, daß der Übergang in fernen Infrarot oder im Mikrowellengebiet zu beobachten ist.

${ }^{4}$ Zum Beispiel E. B. Wilson jr., J. C. Decius u. P. C. Cross, Molecular Vibrations, McGraw Hill Book Co. Inc., New York 1955. 
Potential durch parabolische (harmonische) Potentiale annähern läßt. Der für die Torsionen wesentliche Tunneleffekt infolge der endlich hohen Potentialbarrieren geht dabei schon im Ansatz verloren.

Wenn auch in den Spektren im fernen IR, die den Torsionen zugeschrieben werden, die vom Tunneleffekt erzeugte Aufspaltung von Banden noch nicht genügend sicher beobachtet wurde, so ist sie doch bei höherem Auflösungsvermögen der Spektrographen sicher zu erwarten. Ohne den Tunneleffekt wäre die Torsionsfeinstruktur in den Rotationsspektren unverständlich.

In dieser Arbeit gebe ich die Erweiterung auf den Fall, daß das Molekül zwei äquivalente Methylgruppen besitzt. Das Modell hat also jetzt fünf Freiheitsgrade. Diese Erweiterung scheint mir nützlich, da schon eine Anzahl solcher Moleküle im fernen IR untersucht wurde. Weiter läßt sich bei diesem Molekültyp leichter experimentell feststellen, ob der vorgeschlagene Mechanismus einen wesentlichen Beitrag zur Aktivität der Torsionen liefert. Außerdem ist ein Vergleich mit den von MöLLER ${ }^{5}$ abgeleiteten Auswahlregeln möglich.

Ich beschränke mich auf Moleküle mit der Konfigurationssymmetrie $\mathrm{C}_{2 \mathrm{v}}$, etwa $\left(\mathrm{CH}_{3}\right)_{2} \mathrm{~S}$. Der HamiLToN-Operator lautet in der Formulierung der PAMMethode $^{6}$ :

$$
\begin{aligned}
& H=F p_{1}^{2}+\frac{1}{2} V_{3}\left(1-\cos 3 \alpha_{1}\right) \\
& +F p_{2}^{2}+\frac{1}{2} V_{3}\left(1-\cos 3 \alpha_{2}\right) \\
& +V_{12} \cos 3 \alpha_{1} \cos 3 \alpha_{2}+V_{12}^{\prime} \sin 3 \alpha_{1} \sin 3 \alpha_{2} \\
& +F^{\prime}\left(p_{1} p_{2}+p_{2} p_{1}\right) \quad\left(H_{\mathrm{TT}}\right) \\
& +B_{x} P_{x}^{2}+C P_{y}^{2}+A_{z} P_{z}^{2} \\
& -2 Q_{x} P_{x}\left(p_{1}+p_{2}\right)-2 Q_{z} P_{z}\left(p_{1}-p_{2}\right) . \quad\left(H_{\mathrm{RT}}\right)
\end{aligned}
$$

Die Invarianzgruppe des Hamilton-Operators ist $\mathrm{C}_{3 \mathrm{v}}{ }^{-} \otimes \mathrm{C}_{3 \mathrm{v}}{ }^{+7}$. Die Eigenfunktionen von $H$ lassen sich als Linearkombinationen von Produkten der Eigenfunktionen $U_{\left(v_{1}\right) \sigma_{1}\left(v_{2}\right) \sigma_{2}}\left(\alpha_{1}, \alpha_{2} ;[\gamma]\right)$ des Teiloperators $H_{\mathrm{T}}+H_{\mathrm{TT}}$ und $r_{J K_{-} K_{+}}\left(\psi, \vartheta, \varphi ;\left[\gamma^{\prime}\right]\right)$ des Teiloperators $H_{\mathrm{R}}$ geben:

$$
\begin{aligned}
& \Psi_{\left(v_{1}\right) \sigma_{1}\left(v_{2}\right) \sigma_{2} J\left(K_{-} K_{+}\right)}\left(\alpha_{1}, \alpha_{2}, \psi, \vartheta, \varphi ;[\Gamma]\right) \\
& \underset{\gamma \otimes \gamma_{\gamma^{\prime}=\Gamma} \boldsymbol{a}_{\sigma_{1} \sigma_{2} J}\left(v_{1}\right)\left(v_{2}\right)\left(K_{-} K_{+}\right)}{\left(v_{1}^{\prime}\right)\left(v_{2}^{\prime}\right) K_{-}^{\prime} K_{+}^{\prime}} U_{\left(v_{1}^{\prime}\right) \sigma_{1}\left(v_{2}^{\prime}\right) \sigma_{2}}\left(\alpha_{1}, \alpha_{2} ;[\gamma]\right) r_{J K_{-}^{\prime} K_{+}^{\prime}}\left(\psi, \vartheta, \varphi ;\left[\gamma^{\prime}\right]\right) .
\end{aligned}
$$

Die Summation erstreckt sich dabei über alle möglichen Produkte von Funktionen, die der gleichen Symmetriespezies $\Gamma$ und einem festen Wert von $\sigma_{1}$, $\sigma_{2}, J$ zugeordnet sind. Welche Produkte $\gamma \otimes \gamma^{\prime}$ dieser Bedingung genügen, gibt Tab. III aus ${ }^{7}$ an. Die Zuordnung zu den Quantenzahlen geben Tab. I aus ${ }^{8}$, Tab. II aus ${ }^{7}$ und Tab. II und Tab. VIII aus ${ }^{9}$ an. Da die einzige Dipolmomenkomponente,

$$
\mu_{x}=\mu_{x}(\psi, \vartheta, \varphi)^{\mathbf{1 0}}
$$

der $\mathrm{A}_{1} \mathrm{~A}_{2}$-Spezies der Gruppe $\mathrm{C}_{3 \mathrm{v}}{ }^{-} \otimes \mathrm{C}_{3 \mathrm{v}}{ }^{+}$angehört, erhält man die bekannten Auswahlregeln.

\begin{tabular}{rl|}
$\mathrm{A}_{1} \mathrm{~A}_{1} \longleftrightarrow \mathrm{A}_{1} \mathrm{~A}_{2} ;$ & $\mathrm{A}_{2} \mathrm{~A}_{1} \longleftrightarrow \mathrm{A}_{2} \mathrm{~A}_{2} ;$ \\
$\mathrm{A}_{1} \mathrm{E} \longleftrightarrow \mathrm{A}_{1} \mathrm{E} ;$ & $\mathrm{A}_{2} \mathrm{E} \longleftrightarrow \mathrm{A}_{2} \mathrm{E} ;$ \\
$\mathrm{EA} \mathrm{A}_{1} \longleftrightarrow \mathrm{EA}_{2} ;$ & $\mathrm{EE} \longleftrightarrow \mathrm{EE}$.
\end{tabular}

Es ist also das Matrixelement $\left\langle\psi^{*}[\Gamma]\left|\mu_{x}\right| \psi\left[\Gamma^{\prime}\right]\right\rangle$ $=0$, wenn $\Gamma$ und $\Gamma^{\prime}$ nicht entsprechend den Auswahlregeln gewählt wurde.

${ }^{5}$ K. D. Möller u. H. G. Andresen, J. Chem. Phys. 37, 1800 [1962].

6 J. D. Swalen u. C. C. Costain, J. Chem. Phys. 31, 1562 [1959].

7 R. J. Myers u. E. B. Wilson JR., J. Chem. Phys. 33, 186 [1960].
Begnügt man sich mit den Näherungsfunktionen $U[\gamma] \cdot r\left[\gamma^{\prime}\right]$, die man einem einfacheren Modell zuschreiben kann, bei dem die Wechselwirkung $H_{\mathrm{RT}}$ entfällt, so ist das Matrixelement

$$
\begin{aligned}
& \left\langle U^{*}[\gamma] r^{*}\left[\gamma^{\prime}\right]\left|\mu_{x}\right| U\left[\gamma^{\prime \prime}\right] r\left[\gamma^{\prime \prime \prime}\right]\right\rangle \\
& \quad=\left\langle U^{*}[\gamma] \mid U\left[\gamma^{\prime \prime}\right]\right\rangle \cdot\left\langle r^{*}\left[\gamma^{\prime}\right]\left|\mu_{x}\right| r\left[\gamma^{\prime \prime \prime}\right]\right\rangle=0
\end{aligned}
$$

wegen der Orthogonalität von $U[\gamma]$. Es sei denn, $U^{*}[\gamma]$ und $U[\gamma]$ gehören dem gleichen Torsionsniveau an. Dann gibt das Matrixelement einen reinen Rotationsübergang. Die Torsion ist aber inaktiv.

Wie im Falle des Moleküls mit einer Methylgruppe zeigt sich, daß Torsions-Rotationsübergänge, die in das Gebiet des fernen IR fallen, durch Wechselwirkung zwischen Torsion und Rotation aktiviert werden. Die Wechselwirkung $H_{\mathrm{TT}}$ zwischen zwei Methyltorsionen führt zu keiner Aktivierung.

In Abb. 1 ist beispielsweise ein Teil des Termschemas für $\sigma_{1}=\sigma_{2}=0$ mit den möglichen Über-

8 L. Pierce, J. Chem. Phys. 34, 498 [1961].

9 G. W. King, A. D. Hainer u. P. C. Cross, J. Chem. Phys. 11, 27 [1943].

10 In dem verwendeten Modell ist $\mu_{x}$ unabhängig von $a_{1}$ und $a_{2}$. Die Wahl des Koordinatensystems erfolgte nach ${ }^{5}$. 
gängen angegeben. Abb. 2 gibt das Termschema der sechs energieniedrigsten Kombinationen $\left(v_{1}\right)+\left(v_{2}\right)$ $\leqq 2$. Dabei wurden ausgedehnte Gebiete von Torsions-Rotationsniveaus durch ein Niveau symbolisiert. Das Schema der Übergänge unterscheidet sich von dem, das MöLler ${ }^{5}$ in Abb. 2 gibt $^{11}$.

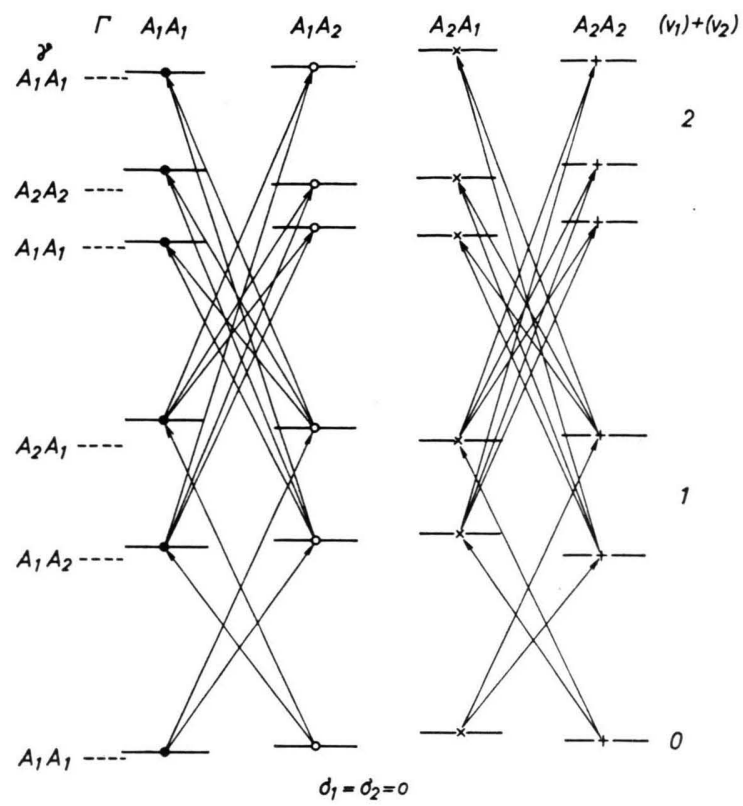

Abb. 1. Ausschnitt aus dem Termschema für $\sigma_{1}=\sigma_{2}=0$ und $\left(v_{1}\right)+\left(v_{2}\right) \leqq 2$. $\gamma$ bezeichnet die Spezies der reinen Torsionszustände *. Darauf aufgebaut sind Torsions-Rotationsniveaus in willkürlicher Anordnung angedeutet. $\bullet \mathrm{A}_{1} \mathrm{~A}_{1}$ $\circ A_{1} A_{2}, \times A_{2} A_{1}^{-},+A_{2} A_{2}$-Spezies.) Die Übergänge $\left(v_{1}\right)+\left(v_{2}\right)=0 \rightarrow 2$ sind nicht eingezeichnet.

Im fernen IR erwartet man also für das in dieser Arbeit behandelte Modell ein Dublett mit dem Abstand $\Delta_{1}$ und in dessen Nachbarschaft ein wegen der geringeren Besetzung schwächeres Triplett mit den Abständen $\Delta_{2}$ und $\Delta_{3}$ gebildet aus Dubletts mit dem Abstand $\Delta_{1}$. Da es sich jeweils um viele Torsions-

11 Für Moleküle mit $\mathrm{C}_{\mathrm{S}}$-Konfigurationssymmetrie tritt der Unterschied nicht auf.

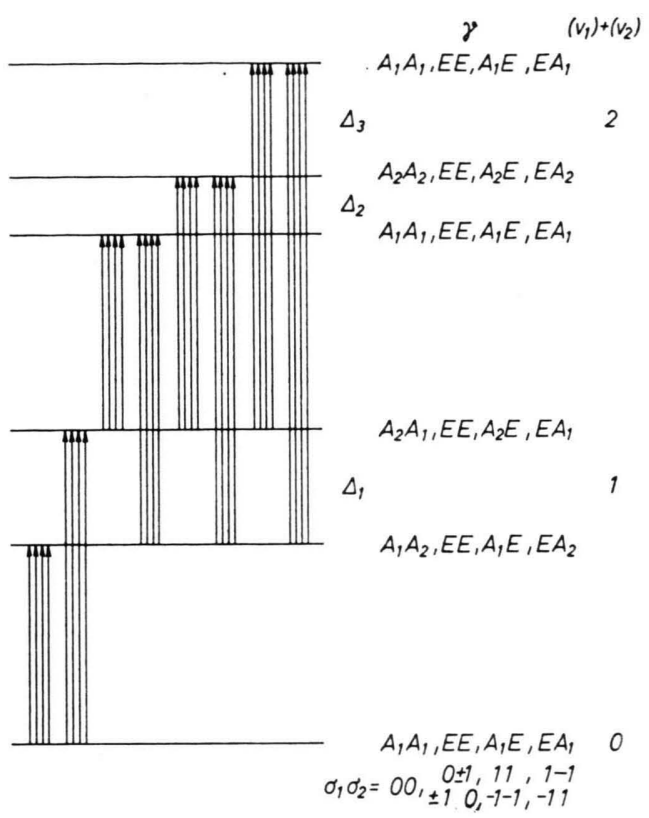

Abb. 2. Ausschnitt aus dem Termschema für $\left(v_{1}\right)+\left(v_{2}\right) \leqq 2$. $\gamma$ bezeichnet die Spezies der reinen Torsionszustände *. Ihre Aufspaltung wurde nicht eingezeichnet. Sie symbolisieren alle diejenigen Torsions-Rotationszustände, bei denen sie den maximalen Mischungsanteil liefern. Diese Zeichnung enthält alle Kombinationen $\sigma_{1} \sigma_{2}$.

Rotationsübergänge handelt, dürften die Multiplettskomponenten schwer auflösbare Banden sein. Nach der bisherigen Kenntnis der Moleküle dieses Typs sollten aber die Abstände $\Delta_{1}$ größer sein als die $\mathrm{Ab}$ stände zwischen den $A_{1} A_{1}$, EE-, $A_{1} E$ - und $E_{1}$-Torsionsniveaus, also experimentell leichter $\mathrm{zu}$ finden sein.

Ich danke Herrn Dr. H. D. Rudolph für Diskussionen, der Deutschen Forschungsgemeinschaft und dem Fonds der Chemischen Industrie für finanzielle Unterstützung.

* Die Reihenfolge innerhalb eines $\left(v_{1}\right)+\left(v_{2}\right)$-Wertes hängt von den Parametern $F^{\prime}, V_{12}, V_{12}^{\prime}$ ab. Die Spingewichte der Torsionsfeinstrukturkomponenten von Rotationslinien angeregter Torsionszustände geben eine Möglichkeit, die Reihenfolge festzulegen, denn die Abnahme der Intensität dieser Rotationslinien mit steigender Torsionsenergie ist im MW-Spektrum deutlich erkennbar. 